\title{
Secondary contouring of flaps
}

\author{
Tae Gon Kim, Man Ki Choi \\ Department of Plastic and Reconstructive Surgery, Yeungnam University College of Medicine, Daegu, Korea
}

Perforator flaps are becoming increasingly common, and as primary thinning techniques are being developed, the need for secondary contouring of flaps is decreasing. However, many reconstructive flap procedures still incorporate secondary debulking to improve the functional and aesthetic outcomes. Direct excision, liposuction, tissue shaving with an arthroscopic cartilage shaver, and skin grafting are the four major methods used for secondary debulking. Direct excision is primarily applied in flaps where the skin is redundant, even though the volume is not excessive. However, due to the limited range of excision, performing a staged excision is recommended. Liposuction can reduce the amount of subcutaneous tissue of the flap and protect the vascular pedicles. However, the main drawback of this method is its limited ability to remove fibrotic tissues, for which the use of a shaver may be more convenient. The main drawback of using a shaver is that it is difficult to simultaneously remove excess skin. Skin grafting enables the removal of sufficient excess tissue to recover the contour of the normal limb and to improve the color match, facilitating excellent aesthetic results.

Keywords Surgical flaps / Perforator flap / Lipectomy / Reoperation / Contouring
Correspondence: Tae Gon Kim Department of Plastic and Reconstructive Surgery, Yeungnam University College of Medicine, 170 Hyeonchung-ro, Nam-gu, Daegu 42415, Korea

Tel: $+82-53-620-3480$

Fax: +82-53-626-0705

E-mail: kimtg0919@daum.net

This work was supported by the 2018 Yeungnam University Research Grant.

Received: 26 May 2018 • Revised: 4 Jul 2018 • Accepted: 5 Jul 2018

pISSN: 2234-6163 • elSSN: 2234-6171 • https://doi.org/10.5999/aps.2018.00542 • Arch Plast Surg 2018;45:319-324

\section{INTRODUCTION}

The advent of the perforator flap technique has significantly improved the aesthetic results in reconstructed areas by enabling the use of relatively thin flaps. The need for secondary contouring of flaps is decreasing with the widespread use of superficial circumflex iliac artery perforator, thoracodorsal artery perforator, and medial sural perforator flaps, which involve anatomically thin subcutaneous fat layers $[1,2]$. Using a primary thinnedout fasciocutaneous flap that does not require secondary contouring may yield good results both functionally and aesthetically in the reconstruction of head, neck, and extremity areas, while the long operating time and the possible instability of blood flow in the flap are considered the main risks [3]. In some cases where the contour appears good immediately postsurgery thanks to the thin flap, it may later look bulkier as the round border of the flap contracts with time. For this reason, many flap operations still depend on secondary debulking to improve the functional and aesthetic outcomes. This is particularly true when (1) the area that is operated on originally has thin skin, such as the hand, the anterior tibial side of the lower leg, ankle, foot dorsum, sole, and the head and neck area; (2) the flap is a thick musculocutaneous flap; and (3) primary thinning of the flap could not be sufficiently performed during the first procedure in a way that would ensure adequate blood flow in the flap [1]. The four major methods used for secondary contouring are direct excision, liposuction, tissue shaving with an arthroscopic cartilage shaver, and skin grafting. This paper reviews the characteristics, advantages, and disadvantages of each method.

\section{DIRECT EXCISION}

Direct excision can primarily be applied in flaps where the volume is not excessive but the skin is redundant. In such cases, li- 
posuction is generally performed, but fat or muscles may also be partially excised if the flap has excessive subcutaneous tissue or muscle tissue. If the flap tissue is excised too early after the operation, the risk of necrosis of the flap increases. The excision of pedicles or perforators must be done carefully, as this may also cause necrosis of the flap. The authors recommend direct excision of redundant flap tissue with incision of the flap along the long axis. It is not recommended to perform incision on the tip of the flap as a $\mathrm{U}$ or $\mathrm{V}$ shape. If excision is necessary in multiple directions, a staged excision is recommended, with an interval of at least 6 months between each procedure (Fig. 1).

\section{LIPOSUCTION}

Liposuction should be performed after all wounds and edema from the flap operation have subsided to avoid excessive correction. Liposuction is safe to perform after a minimum of 3 months postoperatively and when the flap is viable without its main vascular pedicles $[1,4]$. However, liposuction around the pedicles must be performed with care, as reports have described flap necrosis due to damaged vascular pedicles as long as 3 months after the operation [5]. Tumescent fluid containing epinephrine is injected preoperatively to reduce bleeding. The fluid

Fig. 1. Contouring of flap by direct excision

A patient underwent an anterolateral thigh (ALT) free flap operation to cover an open wound caused by the excision of malignant melanoma on the right heel. (A) Gross picture 6 months after performing the ALT free flap operation. The patient complained of discomfort wearing shoes due to the bulky flap, as well as aesthetic dissatisfaction. (B) Direct excision was performed for secondary debulking. The flap was excised along the long axis and subcutaneous tissue was directly excised. (C) Sufficient subcutaneous tissue was removed to improve the contour of the flap, leaving $5 \mathrm{~mm}$ of the tissue just under the flap to prevent necrosis and depression of the skin. (D) Gross picture 1.5 months after the secondary debulking. The patient no longer complained of functional difficulties and the area looked aesthetically satisfactory.
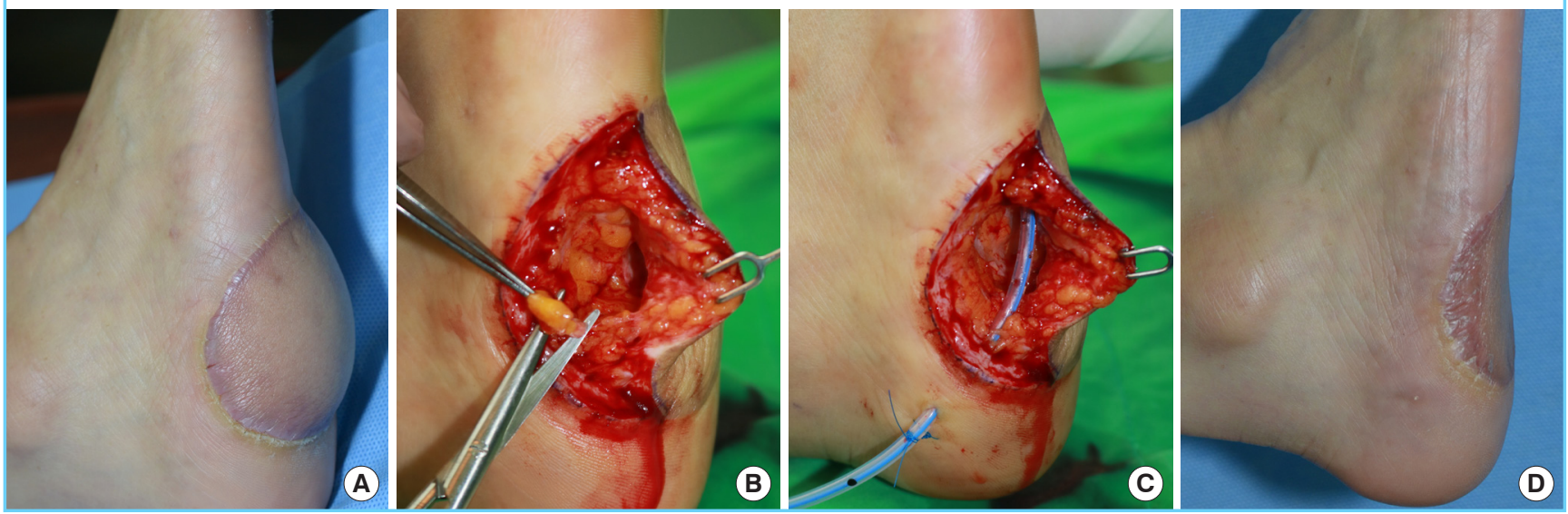

Fig. 2. Injection of tumescent solution prior to liposuction

A patient underwent an anterolateral thigh (ALT) free flap operation to cover an open fracture on the right tibia. (A) Gross picture 6 months after the ALT free flap operation was performed. The bulky flap did not meet aesthetic standards. (B) Picture taken after the injection of tumescent fluid around the flap for secondary debulking using liposuction. The fluid must be injected evenly throughout the subcutaneous fat layer of the flap, until the contour of the flap starts hardening and the color of the skin starts blanching. (C) Gross picture after secondary debulking was performed, showing an aesthetically excellent result.
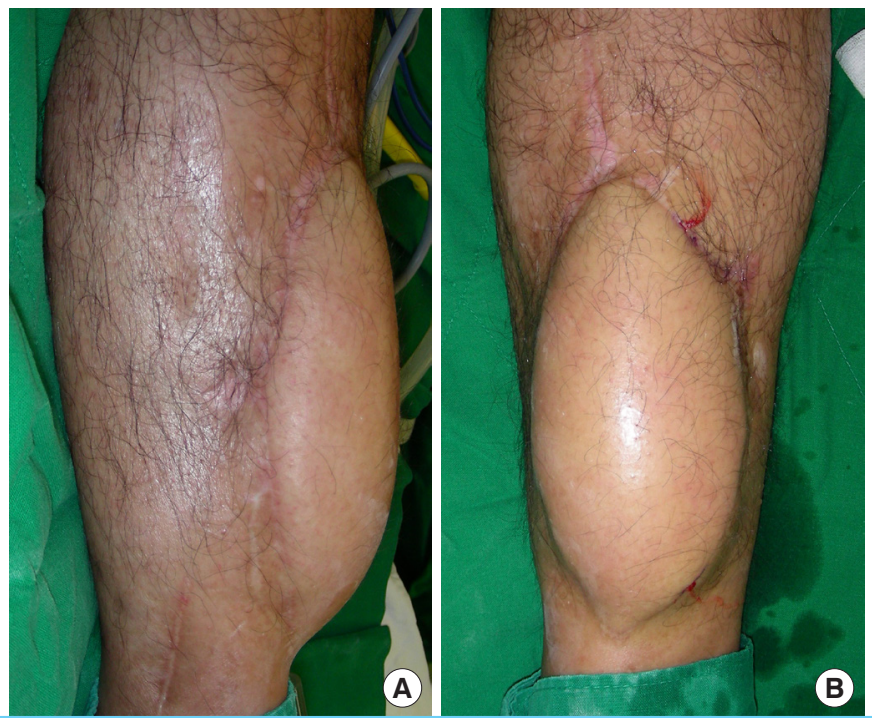
must be injected evenly throughout the subcutaneous fat layer of the flap, until the contour of the flap starts to harden and the color of the skin starts to become blanched (Fig. 2) [1]. Liposuction must avoid the pedicle area and the anastomotic site. Prominent excessive skin after liposuction should be excised partially around the margin of the flap so that the blood flow from the periphery is not blocked (Fig. 3) [6,7]. Such an excision may be performed after waiting a couple of months postsurgery to see if the contour improves naturally from contraction of the skin, rather than performing the excision immediately after liposuction [5]. Liposuction can be safely performed when secondary debulking is planned with an adipofascial flap and skin graft, or with a local flap, such as a distally-based sural flap. Ultrasound-assisted liposuction causes less damage to the tissue and blood flow from negative pressure and mechanical stimulation, although it may cause damage due to the heating effect (Fig. 4) [1,8,9]. The advantages of flap contouring through liposuction are reducing the amount of subcutaneous tissue throughout the flap and protecting the vascular pedicles [1]. It could also maintain dermal blood flow and help manage the contouring of the surrounding tissues. The drawback of this method is its limited ability to remove highly fibrotic tissues $[1,10]$. Gentle compressive dressing or 3 to 5 days of suction draining should be performed postoperatively to prevent seroma and hematoma.

\section{Fig. 3. Excessive liposuction may cause skin necrosis}

A patient underwent a superficial circumflex iliac artery perforator (SCIP) free flap operation to cover an open wound on the right foot dorsum caused by a burn. (A) Gross picture taken 6 months after the SCIP free flap operation. The bulky flap limited dorsiflexion and caused discomfort wearing shoes. (B) Gross picture taken 6 months after secondary debulking using power-assisted liposuction. Partial skin necrosis was seen after the debulking procedure, but recovered without an additional procedure.
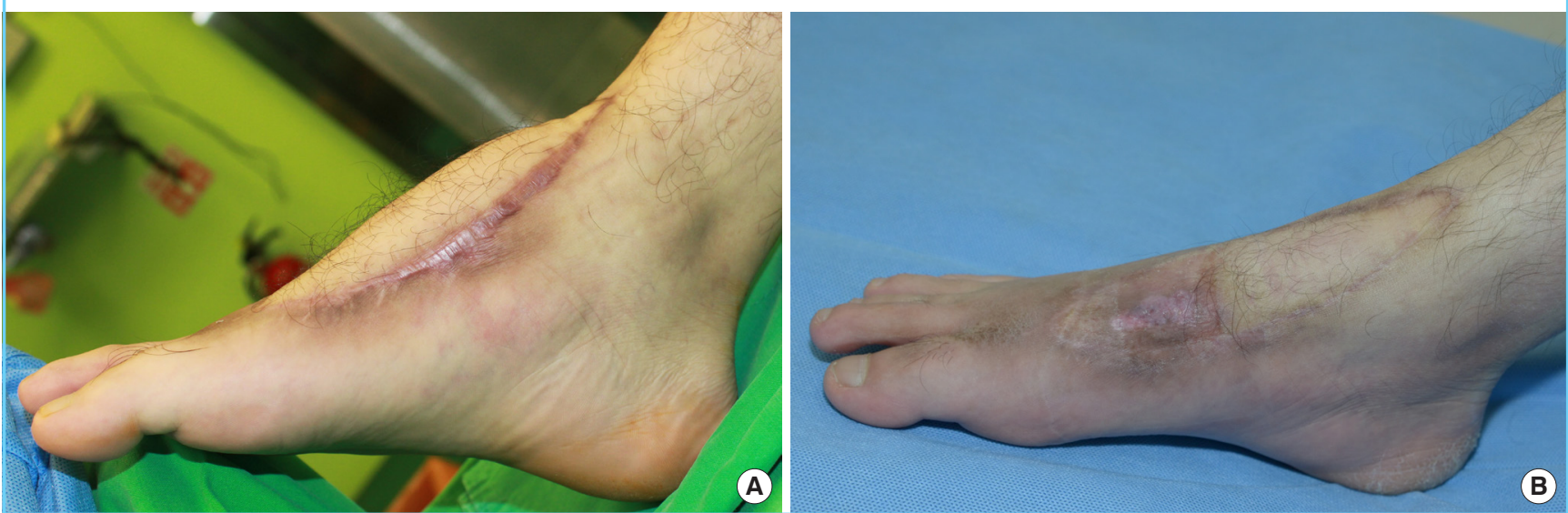

\section{Fig. 4. Flap contouring by ultrasound-assisted liposuction}

A patient underwent anterolateral thigh (ALT) free flap surgery to cover an open wound that occurred due to skin necrosis on the right foot dorsum after cellulitis. (A) Gross picture taken 6 months after ALT free flap surgery. The patient complained of discomfort when wearing shoes due to the bulky flap. (B) Gross picture 15 days after secondary debulking using ultrasound-assisted liposuction. It was safe to excise only one side of the long axis after liposuction.
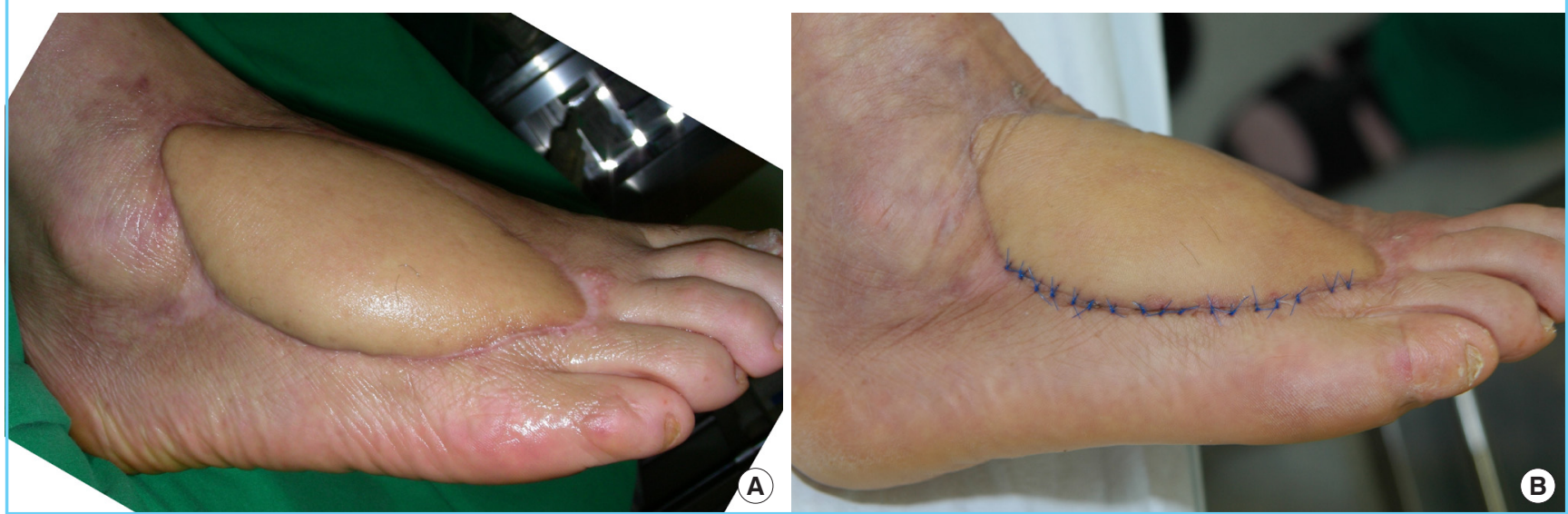


\section{TISSUE SHAVING WITH ARTHROSCOPIC CARTILAGE SHAVER}

Tissue shaving with an arthroscopic cartilage shaver may be more convenient than liposuction for removing fibrotic tissues $[11,12]$. This method is particularly useful on head and neck areas treated with radiotherapy. When contouring the flap with a shaver, 3-5 mm of subcutaneous tissue should be left to prevent bleeding, skin necrosis, and depressions [11]. Operating in the

\section{Fig. 5. Arthroscopic cartilage shaver}
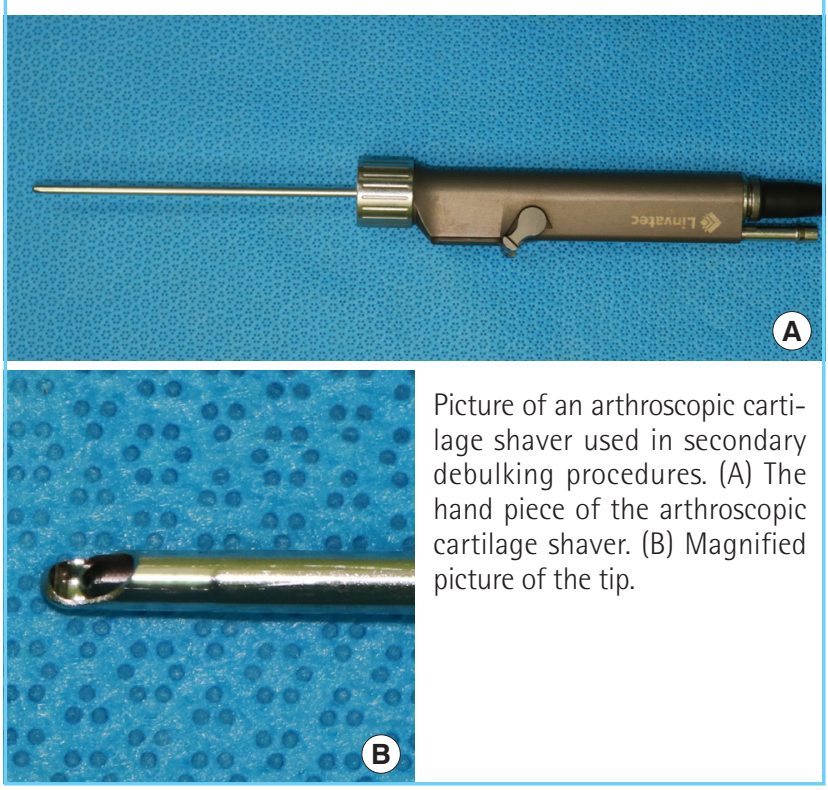

oscillating mode is more convenient than doing so in the rotating mode, and the tip of the shaver should face downward during the operation [3]. Cutting the pedicle area should be avoided to prevent hemorrhage. The drawback of this method is that it is difficult to remove excessive skin at the same time. Removing the skin simultaneously is highly likely to lead to skin necrosis, as hypodermic microvessels are removed together. The excessive skin may contract during the healing process, so excision is recommended if the redundant skin is still visible 3 weeks after shaving (Fig. 5) [11,12].

\section{SIIN GRAFTING}

Common complications after free flap operations include excessive flap volume, inconsistent skin color, reduced flexibility of the skin surface, and reduced sensation of the skin. In theory, a free flap can be as thin as 3-4 mm, but that is not always possible. Secondary measures such as staged excision and liposuction may resolve these complications to a certain degree, but successful results may not be possible in a single-stage operation. Liposuction and excision require leaving at least $5 \mathrm{~mm}$ of tissue to avoid necrosis of the flap, which may seem thick in areas such as the head, ankles, and dorsum of the foot. Another way to cut down on excess in the flap is to remove all layers of skin from the flap, followed by removing the fat or muscle tissues under the flap and re-grafting the skin of the flap, which can be done in a single stage $[13,14]$.

Direct excision of the subcutaneous tissue and fat from the fascial layer should be performed after applying a tourniquet and

Fig. 6. Flap contouring by full-thickness skin graft

A patient underwent an anterolateral thigh (ALT) free flap procedure to cover an open wound caused by skin necrosis on the right foot dorsum after trauma. (A) Gross picture taken 6 months after ALT free flap surgery. The patient complained of discomfort when wearing shoes due to the bulky flap. (B) Debulking was performed after harvesting full-thickness skin from the flap. The vascularity of the recipient bed was confirmed by checking for the presence of numerous pinpoint bleeding spots on the debulked flap area. (C) Gross picture taken 6 months after secondary debulking using a fullthickness skin graft. The contouring can be seen to have improved, and the patient no longer complained of functional discomfort.
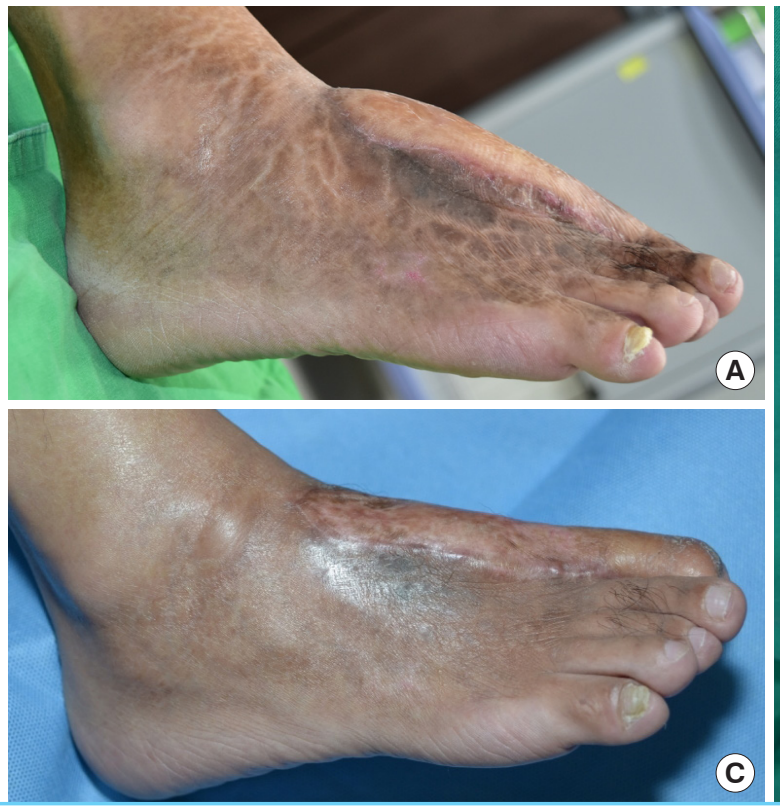

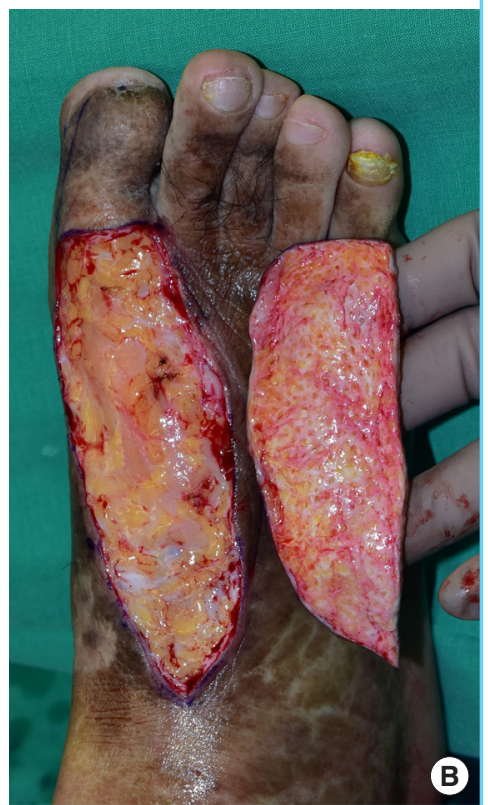


Table. 1. Advantages and disadvantages of methods used for secondary contouring

\begin{tabular}{|c|c|c|}
\hline Method & Advantage & Disadvantage \\
\hline Direct excision & Useful when volume is not excessive and skin is redundant & Risk of flap necrosis \\
\hline Liposuction & $\begin{array}{l}\text { Reduces the amount of subcutaneous tissue throughout the flap } \\
\text { and protects the vascular pedicles }\end{array}$ & Limited ability to remove highly fibrotic tissues \\
\hline $\begin{array}{l}\text { Tissue shaving with an arthroscopic cartilage } \\
\text { shaver }\end{array}$ & More convenient than liposuction for removing fibrotic tissues & Difficult to remove excessive skin at the same time \\
\hline Skin grafting & Allows removal of excessive tissue in a single-stage operation & Necrosis of skin \\
\hline
\end{tabular}

harvesting full-thickness skin from the flap. Tangential excision is performed on the muscular part of the myocutaneous flap, if necessary. The tourniquet is loosened to confirm the vascularity of the recipient bed through the presence of numerous pinpoint bleeding spots in the debulked flap area. Vascular pedicles can be well preserved if the fascial layer is included in the flap when it is elevated, and if the fascia is left in place after removing subcutaneous tissues [15]. Grafting of the harvested full-thickness skin onto the raw surface of the fascia is performed after hemostasis. The full-thickness skin grafting procedure enables the sufficient removal of excess tissue to recover the contour of the normal limb and to improve the color match, enabling excellent aesthetic results. The skin also maintains its softness and pliability, as the flap preserves the fascial layer [16]. Sensory outcomes, as checked by 2-point discrimination, have also reported to be enhanced (Fig. 6).

\section{CONCLUSIONS}

Functional and aesthetic issues may arise due to the thickness of the flap after reconstructive flap surgery, and secondary debulking can contribute to improved outcomes. This paper has suggested the use of four of the many methods of secondary contouring (Table 1). Using a thin flap in the first place may be ideal for the operation, but when impossible, secondary flap debulking can enhance both functional and aesthetic outcomes.

\section{NOTES}

\section{Conflict of interest}

No potential conflict of interest relevant to this article was reported.

\section{Ethical approval}

The study was performed in accordance with the principles of the Declaration of Helsinki.

\section{Patient consent}

The patients provided written informed consent for the publica- tion and the use of their images.

\section{ORCID}

Tae Gon Kim https://orcid.org/0000-0002-6738-4630

\section{REFERENCES}

1. Kim TG, Hong JP, Chung YK. Clinical experience of countouring fasciocutaneous flap using ultrasound assisted liposuction.J Korean Microsurg Soc 2003;12:99-104.

2. Hallock GG. Defatting of flaps by means of suction-assisted lipectomy. Plast Reconstr Surg 1985;76:948-52.

3. Karakullukcu B, van Laarhoven CM, Smeele LE, et al. Functional and aesthetic recontouring of free flap reconstructions of the head and neck region with microdebrider. Kulak Burun Bogaz Ihtis Derg 2014;24:118-22.

4. Wooden WA, Shestak KC, Newton ED, et al. Liposuctionassisted revision and recontouring of free microvascular tissue transfers. Aesthetic Plast Surg 1993;17:103-7.

5. Ibrahim AE, Janom H, Raad M. Liposuction contouring after head and neck free flap reconstruction. Anaplastology 2015;4:145.

6. Hallock GG. Conventional liposuction-assisted debulking of muscle perforator flaps. Ann Plast Surg 2004;53:39-43.

7. Hallock GG. Liposuction for debulking free flaps. J Reconstr Microsurg 1986;2:235-9.

8. Reuben CM, Bastidas N, Sharma S. Power-assisted suction lipectomy of fasciocutaneous flaps in the extremities. Ann Plast Surg 2010;65:60-5.

9. Yamanaka K, Ichikawa T, Horiuchi Y. Flap defatting with an ultrasonic surgical aspirator. Plast Reconstr Surg 1997;99: 888-91.

10. Askouni EP, Topping A, Ball S, et al. Outcomes of anterolateral thigh free flap thinning using liposuction following lower limb trauma. J Plast Reconstr Aesthet Surg 2012;65:474-81.

11. Tan NC, Cigna E, Varkey P, et al. Debulking of free myocutaneous flaps for head and neck reconstruction using an arthroscopic shaver. Int J Oral Maxillofac Surg 2007;36:450-2.

12. Cigna E, Sassu P, Varkey P, et al. Debulking of free perforator 
flaps for head and neck reconstruction using an arthroscopic shaver. Ann Plast Surg 2005;55:441.

13. Lin TS, Jeng SF. Full-thickness skin graft as a one-stage debulking procedure after free flap reconstruction for the lower leg. Plast Reconstr Surg 2006;118:408-12.

14. Lin TS. One-stage debulking procedure after flap reconstruction for degloving injury of the hand. J Plast Reconstr
Aesthet Surg 2016;69:646-51.

15. Lin TS, Quing R. Long-term results of a one-stage secondary debulking procedure after flap reconstruction of the foot. Plast Reconstr Surg 2016;138:923-30.

16. Lin TS, Jeng SF, Chiang YC. Resurfacing with full-thickness skin graft after debulking procedure for bulky flap of the hand. J Trauma 2008;65:123-6. 